1 Hacettepe Journal of Mathematics and Statistics

$\bigcap$ Volume 47 (4) (2018), 835-843

\title{
Some properties of the total graph and regular graph of a commutative ring
}

\author{
Manal Ghanem* and Khalida Nazzal ${ }^{\dagger}$
}

\begin{abstract}
Let $R$ be a commutative ring with unity. The total graph of $R, T(\Gamma(R))$, is the simple graph with vertex set $R$ and two distinct vertices are adjacent if their sum is a zero-divisor in $R$. Let $\operatorname{Reg}(\Gamma(\mathrm{R}))$ and $Z(\Gamma(R))$ be the subgraphs of $T(\Gamma(R))$ induced by the set of all regular elements and the set of zero-divisors in $R$, respectively. We determine when each of the graphs $T(\Gamma(R)), \operatorname{Reg}(\Gamma(R))$, and $Z(\Gamma(R))$ is locally connected, and when it is locally homogeneous. When each of $\operatorname{Reg}(\Gamma(R))$ and $Z(\Gamma(R))$ is regular and when it is Eulerian.
\end{abstract}

Keywords: Total graph of a commutative ring, Regular graph of a commutative ring, Locally connected, Locally homogeneous, Regular graph, Eulerian graph.

Mathematics Subject Classification (2010): 13A15, 05C99

Received : 31.05.2016 Accepted : 12.06.2017 Doi : 10.15672/HJMS.2017.490

\section{Introduction}

Throughout this paper $R$ will be used to denote a commutative ring with unity $1 \neq 0$. Let $Z(R)$ be the set of all zero-divisors of $R$. The total graph of $R$ is the simple graph with vertex set $R$ where two distinct vertices $x$ and $y$ are adjacent if $x+y \in Z(R)$. This graph, denoted by $T(\Gamma(R))$, was introduced by Anderson and Badawi in [1], the authors gave full description for the case when $Z(R)$ is an ideal. On the other hand, they computed some graphical invariants such as the diameter and the girth of $T(\Gamma(R))$. Akbari and et al. [3], proved that if $R$ is a finite ring, then a connected total graph is Hamiltonian. Maimani and et al. [12] investigated the genus of $T(\Gamma(R))$. The radius of $T(\Gamma(R))$ was computed in [13]. The domination number of $T(\Gamma(R))$ is determined independently in both [7] and [16]. For a finite commutative ring $R$, a characterization of

\footnotetext{
*Department of Mathematics, School of Science, The University of Jordan, Amman 11942 Jordan, 
Eulerian $T(\Gamma(R))$ is given in [16]. Minimum zero -sum $k$-flows for $T(\Gamma(R))$ are considered in [15]. The complement of $T(\Gamma(R))$ is investigated in [5]. Vertex-connectivity and edgeconnectivity of $T(\Gamma(R))$, where $\mathrm{R}$ is a finite commutative ring, are discussed in [14]. Some properties of the regular graph $\operatorname{Reg}(\Gamma(R))$ are studied in [4]. The line graph of $T(\Gamma(R))$ is investigated in [8]. Furthermore, the generalized total graph of $R$ is defined in [2]. For a survey on the total graph of a commutative ring, the reader may refer to [6] or [10].

The following theorem gives full description of the graph $T(\Gamma(R))$ when $Z(R)$ is an ideal of $R$.

1.1. Theorem. [1] Let $R$ be a ring such that $Z(R)$ is an ideal of $R$. Let $|Z(R)|=\lambda$, $|R / Z(R)|=\mu$.

(i) If $2 \in Z(R)$, then $\operatorname{Reg}(\Gamma(R))$ is the union of $\mu-1$ disjoint $K_{\lambda}{ }^{\prime} s$.

(ii) If $2 \in \operatorname{Reg}(R)$, then $\operatorname{Reg}(\Gamma(R))$ is the union of $(\mu-1) / 2$ disjoint $K_{\lambda, \lambda}{ }^{\prime} s$.

(iii) $Z(\Gamma(R))$ is the complete graph, $K_{\lambda}$.

(v) $\operatorname{Reg}(\Gamma(R))$ is connected if and only if $R / Z(R) \cong \mathbb{Z}_{2}$ or $R / Z(R) \cong \mathbb{Z}_{3}$.

Several structural properties of $T(\Gamma(R)), \operatorname{Reg}(\Gamma(R))$, and $Z(\Gamma(R))$ will be considered. Section 2 addresses the problems "when is each of the graphs $T(\Gamma(R)), \operatorname{Reg}(\Gamma(R))$, and $Z(\Gamma(R))$ locally connected?". Section 3 answers the problem "when is each of the graphs $\operatorname{Reg}(\Gamma(R))$, and $Z(\Gamma(R))$ regular?". In Section 4, Eulerian $\operatorname{Reg}(\Gamma(R))$, and $Z(\Gamma(R))$ are characterized, where $R$ is a finite commutative ring. Section 5 addresses the problem "when is each of the graphs $T(\Gamma(R)), \operatorname{Reg}(\Gamma(R))$, and $Z(\Gamma(R))$ locally homogeneous?"

\section{When are $T(\Gamma(R)), \operatorname{Reg}(\Gamma(R))$, and $Z(\Gamma(R))$ Locally Connected?}

Let $G$ be a graph with vertex set and edge set $V(G)$ and $E(G)$ respectively. Let $v \in$ $V(G)$, the open neighborhood, $N(v)$, of $v$ is defined by $N(v)=\{u \in V(G): u v \in E(G)\}$. The graph $G$ is said to be locally connected if for all $v \in V(G), N(v)$ induces a connected graph in $G$. Thus, if $G$ is a union of complete graphs, then $G$ is locally connected and if a graph $G$ has a bipartite component, other than $K_{1,1}$, then it is not locally connected. This, together with Theorem 1.1 give the following theorem.

2.1. Theorem. Let $R$ be a ring and $Z(R)$ be an ideal of $R$.

(i) $Z(\Gamma(R))$ is a locally connected graph.

(ii) $\operatorname{Reg}(\Gamma(R))$ and $T(\Gamma(R))$ are locally connected graphs if and only if $2 \in Z(R)$, or $R$ is an integral domain.

The next theorem considers the case when $R$ is a product of two rings.

2.2. Theorem. Let $R$ be a product of two rings $R_{1}$ and $R_{2}$. Then $T(\Gamma(R))$ is locally connected if and only if either $R_{1}$ or $R_{2}$ is not an integral domain.

Proof. First, we study the case when both $R_{1}$ and $R_{2}$ are integral domains. Suppose that $2 \in \operatorname{Reg}(R)$ (i.e. $2 \in \operatorname{Reg}\left(R_{1}\right)$ and $\left.2 \in \operatorname{Reg}\left(R_{2}\right)\right)$, then $(-1,1)$ and $(-1,-1)$ are only adjacent to each other in $N((1,0))$ and hence there is no path between $(-1,0)$ and $(-1,1)$ in $N((1,0))$. If $2 \in Z\left(R_{1}\right)$ and $2 \in \operatorname{Reg}\left(R_{2}\right)$, then $(0,-1)$ is an isolated vertex in $N((1,1))$. And if $2 \in Z\left(R_{1}\right)$ and $2 \in Z\left(R_{2}\right)$, then there is no path joining $(1,0)$ and $(0,1)$ in $N((1,1))$. So, $T(\Gamma(R))$ is not locally connected.

Now, we may assume that either $R_{1}$ or $R_{2}$ is not an integral domain. Let $N_{i}(u)$, denotes the open neighborhood of $u$ in $T\left(\Gamma\left(R_{i}\right)\right)$. Let $(a, b) \in R$ and $(x, y),(z, w) \in$ $N((a, b))$. If $(x, y)$ and $(z, w)$ are non-adjacent in $N((a, b))$, then we have four cases:

Case 1: $x \in N_{1}(a)$ and $w \in N_{2}(b)$ or $\left(z \in N_{1}(a)\right.$ and $\left.y \in N_{2}(b)\right)$.

Assume that $x \in N_{1}(a)$ and $w \in N_{2}(b)$. Then $(x, y)-(a, w)-(x, b)-(z, w)$ is a path in $N((a, b))$. 
Case 2: $x, z \in N_{1}(a)$ or $\left(y, w \in N_{2}(b)\right)$.

Assume that $x, z \in N_{1}(a)$. Then we have three cases.

Case 2.1: $2 \in Z\left(R_{1}\right)$.

Choose $t \in R_{2} \backslash\{b\}$, then $(a, t) \in N((a, b))$. So, $(x, y)-(a, t)-(z, w)$ is a path in $N((a, b))$.

Case 2.2: $2 \in \operatorname{Reg}\left(R_{1}\right)$ and $2 \in Z\left(R_{2}\right)$.

If $R_{1}$ is not an integral domain, then there exist $t, s \in Z\left(R_{1}\right)$ such that $-x+t \neq a$ and $-z+r \neq a$. Then if $-x+t \neq-z+r$, the path $(x, y)-(-x+t, b)-(-z+r, b)-(z, w)$ is obtained. Otherwise, $(x, y)-(-x+t, b)-(z, w)$ is a path in $N((a, b))$. Now, if $R_{2}$ is not an integral domain, then there exists $r \in Z\left(R_{2}\right)$ such that $-b+r \neq b$. So, $(x, y)-(a,-b+r)-(z, w)$ is a path in $N((a, b))$.

Case 2.3: $2 \in \operatorname{Reg}\left(R_{2}\right)$.

If $R_{2}$ is not an integral domain, then there exists $r \in Z\left(R_{2}\right)$ such that $-b+r \neq b$. So, $(x, y)-(a,-b+r)-(z, w)$ is a path in $N((a, b))$. If $R_{1}$ is not an integral domain, then there exist $t, s \in Z\left(R_{1}\right)$ such that $-x+t \neq a$ and $-z+r \neq a$. So, when $-x+t \neq-z+r$, we get the path $(x, y)-(-x+t,-b)-(x, b)-(-z+r,-b)-(z, w)$ in $N((a, b))$. Otherwise, we get the path $(x, y)-(-x+t,-b)-(z, w)$.

Case 3: $x \in N_{1}(a), z \in R_{1}-N_{1}(a)$ and $w=b$ or $\left(x=a, y \in R_{2}-N_{2}(b)\right.$ and $\left.w \in N_{2}(b)\right)$.

Assume that $x \in N_{1}(a), z \in R_{1}-N_{1}(a)$ and $w=b$. Then $2 b \in Z\left(R_{2}\right)$. So, $R_{1}$ is not an integral domain, gives $-x+t \neq a$ for some $t \in Z\left(R_{1}\right)$. Therefore, $(x, y)-(-x+t, b)-(z, w)$ is a path in $N((a, b))$. While $R_{2}$ is not an integral domain, implies that $-b+r \neq b$ for some $r \in Z\left(R_{2}\right)$. So, $(x, y)-(a,-b+r)-(z, w)$ is a path in $N((a, b))$.

Case 4: $x=a, w=b, 2 a \in Z\left(R_{1}\right)$, and $2 b \in Z\left(R_{2}\right)$ or $\left(y=b, x=a, 2 a \in Z\left(R_{1}\right)\right.$ and $\left.2 b \in Z\left(R_{2}\right)\right)$.

Assume that $x=a, w=b, 2 a \in Z\left(R_{1}\right)$, and $2 b \in Z\left(R_{2}\right)$. Then $R_{1}$ is not an integral domain, implies that $-x+t \neq a$ for some $t \in Z\left(R_{1}\right)$ and $R_{2}$ is not an integral domain implies that $-b+r \neq b$ for some $r \in Z\left(R_{2}\right)$. Thus, $(x, y)-(-x+t, b)-(z, w)$ or $(x, y)-(a,-b+r)-(z, w)$ is a path in $N((a, b))$.

If $R$ is a local ring, then $Z(R)$ is an ideal and hence $Z(\Gamma(R))$ is a complete graph which is obviously locally connected. When $R$ is a product of two rings, we have the following theorem.

2.3. Theorem. Let $R$ be a product of two rings $R_{1}$ and $R_{2}$. Then $Z(\Gamma(R))$ is locally connected if and only if either $R_{1}$ or $R_{2}$ is not an integral domain.

Proof. Observe that if $R$ is a product of two integral domains, then there is no path joining $(1,0)$ and $(0,1)$ in $N((0,0))$. So $Z(\Gamma(R))$ is not locally connected. Assume that either $R_{1}$ or $R_{2}$ is not an integral domain. Since $(0,0) \in N((a, b))$ for any non-zero zerodivisors $(a, b)$, we have $(x, y)-(0,0)-(z, w)$ is a path joining $(x, y)$ and $(z, w)$ in $N((a, b))$. Thus $N((a, b))$ is locally connected for all $(a, b) \in Z(R)-\{0\}$. So it remains to study connectivity of the graph induced by $N((0,0))$. Assume that $(x, y)$ and $(z, w)$ are two nonadjacent vertices in $N((0,0))$, then $x \in Z\left(R_{1}\right) \backslash\{0\}$ implies that $(x, y)-(-x,-w)-(z, w)$ is a path in $N((0,0))$ and $y \in Z\left(R_{2}\right) \backslash\{0\}$ implies that $(x, y)-(-z,-y)-(z, w)$ is a path in $N((0,0))$.

Next, we will investigate when $\operatorname{Reg}(\Gamma(R))$ is locally connected. If $R$ is a local ring, then $\operatorname{Reg}(\Gamma(R))$ is locally connected if $R$ is an integral domain or $2 \in Z(R)$. If $R$ is a product of two rings, then we have the following.

2.4. Theorem. Let $R$ be a product of two rings and $2 \in \operatorname{Reg}(R)$. Then $\operatorname{Reg}(\Gamma(R))$ is locally connected. 
Proof. Assume that $(a, b) \in \operatorname{Reg}(R)$ and $(x, y),(z, w)$ are two non-adjacent vertices in $N((a, b))$. Then $x \in N(a)$ gives the path $(x, y)-(a,-b)-(-a,-w)-(z, w)$ in $N((a, b))$, and $y \in N(b)$ gives the path $(x, y)-(-a, b)-(-z,-b)-(z, w)$ in $N((a, b))$.

Let $R=R_{1} \times R_{2}$, then it is easy to see that if $\left|\operatorname{Reg}\left(R_{1}\right)\right|=1$, then $2 \in Z(R)$ and $\operatorname{Reg}(\Gamma(R))$ is a complete graph and hence it is locally connected.

A Boolean ring provides an example of a ring $R$ with only one regular element, this is due to the fact that for all $r \in R, r=r^{2}$. So, we get the following.

2.5. Theorem. If $R$ is a Boolean ring or $R$ is a product of rings with at least one Boolean factor, then $\operatorname{Reg}(\Gamma(R))$ is a complete graph.

At this point it makes sense to require that $\left|\operatorname{Reg}\left(R_{i}\right)\right| \geq 2$, for all $i$.

2.6. Theorem. Let $R$ be a product of two local rings $R_{1}$ and $R_{2}$ such that $2 \in Z(R)$ and $\left|\operatorname{Reg}\left(R_{i}\right)\right| \geq 2$ for $i=1,2$. Then $\operatorname{Reg}(\Gamma(R))$ is locally connected if and only if $R_{1}$ or $R_{2}$ is not an integral domain.

Proof. Suppose that $R=R_{1} \times R_{2}$ where $R_{1}$ and $R_{2}$ are integral domains, $2 \in Z(R)$ and $\left|\operatorname{Reg}\left(R_{i}\right)\right| \geq 2$ for $i=1,2$. Choose $(t, s) \in \operatorname{Reg}(R) \backslash\{(1,1)\}$, then $2 \in Z\left(R_{1}\right)$ and $2 \in Z\left(R_{2}\right)$ imply that $(1, s)$ and $(t, 1)$ are two non-adjacent vertices in $\operatorname{Reg}(\Gamma(R))$ and there is no path joining them in $N((1,1))$. If $2 \in Z\left(R_{1}\right)$ and $2 \in \operatorname{Reg}\left(R_{2}\right)$, then $(1,-1)$ and $(t,-1)$, where $t \in \operatorname{Reg}\left(R_{1}\right) \backslash\{1\}$, are non-adjacent vertices in $N((1,1))$, with no path joining them in $N((1,1))$. So $\operatorname{Reg}(\Gamma(R))$ is not locally connected.

Conversely, let $R=R_{1} \times R_{2}$ where $R_{1}$ and $R_{2}$ are two local rings such that $2 \in Z(R)$ and $\left|\operatorname{Reg}\left(R_{i}\right)\right| \geq 2$, for $i=1,2$. Without loss of generality, assume that $2 \in Z\left(R_{1}\right)$. Let $(a, b) \in \operatorname{Reg}(R)$ and $(x, y),(z, w)$ be two non-adjacent vertices in $N((a, b))$. If $R_{1}$ is not an integral domain, then there exists $t \in Z\left(R_{1}\right)$ such that $t+a \neq a$. Since $Z\left(R_{1}\right)$ is an ideal of $R, t+a \in \operatorname{Reg}\left(R_{1}\right)$. Therefore, $(x, y)-(a+t,-y)-(a+t,-w)-(z, w)$ is a path in $N((a, b))$. And if $R_{2}$ is not an integral domain, then $t-y \neq b$ and $s-w \neq b$ for some $t, s \in Z\left(R_{2}\right)$, so $(x, y)-(a, t-y)-(a, s-w)-(z, w)$ is a path in $N((a, b))$ when $t-y \neq s-w$, otherwise, we have the path $(x, y)-(a, t-y)-(z, w)$ in $N((a, b))$.

2.7. Theorem. If $R=\prod_{i=1}^{n} R_{i}, n \geq 3$, then $\operatorname{Reg}(\Gamma(R))$ is locally connected.

Proof. Let $a=\left(a_{i}\right) \in \operatorname{Reg}(R)$ and $u=\left(u_{i}\right)$ and $v=\left(v_{i}\right)$ be two non-adjacent vertices in $N(a)$. Since $u \in N(a), a_{i}+u_{i} \in Z\left(R_{i}\right)$, for some $i$, say for $i=1$. Define $w=\left(w_{i}\right)$ such that $w_{1}=u_{1}, w_{2}=-u_{2}, w_{3}=-v_{3}$ and $w_{i}=1$ for all $i \geq 4$, then $u-w-v$ is a path in $N(a)$.

An Artinian ring is a ring that satisfies the descending chain condition on ideals. An Artinian ring $R$ can be written uniquely (up to isomorphism) as a finite direct product of Artinian local rings. Since $Z(R)$ is an ideal of $R$ when $R$ is local, we may conclude the following.

2.8. Theorem. Let $R$ be an Artinian ring, then

(i) $T(\Gamma(R))$ is not locally connected if and only if $R$ is a local ring satisfying $2 \in$ $\operatorname{Reg}(R)$ and $R$ is not an integral domain or $R$ is a product of integral domains.

(ii) $Z(\Gamma(R))$ is not locally connected if and only if $R$ is a product of two integral domains.

(iii) $\operatorname{Reg}(\Gamma(R))$ is not locally connected if and only if $R$ is a local ring satisfying $2 \in \operatorname{Reg}(R)$ and $R$ is not an integral domain or $R=R_{1} \times R_{2}, 2 \in Z(R)$, and $\left|\operatorname{Reg}\left(R_{i}\right)\right| \geq 2$ and $R_{i}$ is an integral domain for $i=1,2$. 
2.9. Corollary. (i) $T\left(\Gamma\left(\mathbb{Z}_{n}\right)\right)$ is not locally connected if and only if $n=t^{m}$, where $t$ is an odd prime and $m \geq 2$ or $n=t_{1} t_{2}$, where $t_{1}$, and $t_{2}$ are distinct primes.

(ii) $Z\left(\Gamma\left(\mathbb{Z}_{n}\right)\right)$ is not locally connected if and only if $n=t_{1} t_{2}$ where $t_{1}$ and , $t_{2}$ are two distinct primes.

(iii) $\operatorname{Reg}\left(\Gamma\left(\mathbb{Z}_{n}\right)\right)$ is not locally connected if and only if $n=t^{m}$, where $t$ is an odd prime and $m \geq 2$.

\section{When are $T(\Gamma(R)), \operatorname{Reg}(\Gamma(R))$, and $Z(\Gamma(R))$ regular?}

In this section, we study regularity of the graphs $T(\Gamma(R)), \operatorname{Reg}(\Gamma(R))$, and $Z(\Gamma(R))$ for any ring $R$. Maimani et al. [12] proved that in $T(\Gamma(R)), \operatorname{deg}(u)=|Z(R)|-1$ if $2 \in Z(R)$ or $u \in Z(R)$, and $\operatorname{deg}(u)=|Z(R)|$ otherwise. So, $T(\Gamma(R))$ is regular graph only if $2 \in Z(R)$ or $R$ is an infinite non integral domain ring.

Now, we examine regularity of $\operatorname{Reg}(\Gamma(R))$. Clearly, if $Z(R)$ is an ideal, then $\operatorname{Reg}(\Gamma(R))$ is regular of degree $|Z(R)|-1$, when $2 \in Z(R)$ and it is regular graph of degree $|Z(R)|$ when $2 \in \operatorname{Reg}(R)$.

The following theorems address the case when $R$ is a product of two rings.

3.1. Theorem. Let $R$ be a product of two rings $R_{1}$ and $R_{2}$ where $R_{1}$ and $R_{2}$ are two rings such that $\left|\operatorname{Reg}\left(R_{1}\right)\right|=n_{1}$ and $\left|\operatorname{Reg}\left(R_{2}\right)\right|=n_{2}$. Let $\left(u_{1}, u_{2}\right) \in \operatorname{Reg}(R)$ and $\operatorname{deg}_{1}\left(u_{1}\right)=r_{1}$ and $\operatorname{deg}_{2}\left(u_{2}\right)=r_{2}$, where $\operatorname{deg}_{i}\left(u_{i}\right)$ is the degree of $u_{i}$ in $\operatorname{Reg}\left(\Gamma\left(R_{i}\right)\right)$. Then the degree of the vertex $\left(u_{1}, u_{2}\right)$ in $\operatorname{Reg}(\Gamma(R))$ is given by,

$$
\operatorname{deg}\left(\left(u_{1}, u_{2}\right)\right)= \begin{cases}n_{2} r_{1}+n_{1} r_{2}-r_{1} r_{2}, & \text { if } 2 \in \operatorname{Reg}(R) ; \\ n_{1} r_{2}+n_{2} r_{1}+\left(n_{1}+n_{2}\right)-\left(r_{1}+r_{2}\right)-r_{1} r_{2}-2, & \text { if } 2 \in Z\left(R_{1}\right) \text { and } 2 \in Z\left(R_{2}\right) ; \\ n_{1} r_{2}+n_{2} r_{1}-r_{2}+n_{2}-r_{1} r_{2}-1, & \text { if } 2 \in Z\left(R_{1}\right) \text { and } 2 \in \operatorname{Reg}\left(R_{2}\right) .\end{cases}
$$

Proof. Note that if $2 \in \operatorname{Reg}(R)$, then $N\left(\left(u_{1}, u_{2}\right)\right)=\left\{(a, b) \in \operatorname{Reg}(R): a \in N\left(u_{1}\right)\right.$ or $b \in$ $\left.N\left(u_{2}\right)\right\}$. So, $\left|N\left(\left(u_{1}, u_{2}\right)\right)\right|=r_{1} n_{2}+n_{1} r_{2}-r_{1} r_{2}$. If $2 \in Z\left(R_{1}\right)$ and $2 \in Z\left(R_{2}\right)$, then $N\left(\left(u_{1}, u_{2}\right)\right)=\left\{(a, b) \in \operatorname{Reg}(R) \backslash\left\{\left(u_{1}, u_{2}\right)\right\}: a \in N\left(u_{1}\right) \cup\left\{u_{1}\right\}\right.$ or $\left.b \in N\left(u_{2}\right) \cup\left\{u_{2}\right\}\right\}$. So, $\left|N\left(\left(u_{1}, u_{2}\right)\right)\right|=\left(r_{2}+1\right) n_{1}+\left(r_{1}+1\right) n_{2}-\left(r_{1}+1\right)\left(r_{2}+1\right)-1$. If $2 \in Z\left(R_{1}\right)$ and $2 \in \operatorname{Reg}\left(R_{2}\right)$, then $N\left(\left(u_{1}, u_{2}\right)\right)=\left\{(a, t) \in \operatorname{Reg}(R) \backslash\left\{\left(u_{1}, u_{2}\right)\right\}: a \in N\left(u_{1}\right) \cup\left\{u_{1}\right\}\right.$ or $\left.b \in N\left(u_{2}\right)\right\}$. So, $\left|N\left(\left(u_{1}, u_{2}\right)\right)\right|=\left(r_{1}+1\right) n_{2}+n_{1} r_{2}-\left(r_{1}+1\right) r_{2}-1$.

Since for any local ring $R$ the graph $\operatorname{Reg}(\Gamma(R))$ is regular and every finite ring is a product of local rings by using Theorem 3.1 we get the following.

3.2. Theorem. If $R$ is a finite ring, then $\operatorname{Reg}(\Gamma(R))$ is a regular graph.

The following two lemmas will be useful in the subsequent work.

3.3. Lemma. Let $R$ be a finite ring. Then

(i) if $|R|$ is even, then $|Z(R)|$ and $|\operatorname{Reg}(R)|$ are both odd when $R$ is a field or a product of fields of even orders, and they are both even otherwise.

(ii) if $|R|$ is odd, then $|\operatorname{Reg}(R)|$ is even and $|Z(R)|$ is odd.

If $R$ is a ring, then $2 \in Z(R)$ if and only if $|r|=2$ in $(R,+)$, for some $r \in R \backslash\{0\}$. If $R$ is a finite ring, then $2 \in Z(R)$ if and only if $|R|$ is even.

Using Theorem 3.1 and the same notation, it is easy to conclude the following.

3.4. Lemma. Let $R$ be a product of two local rings $R_{1}$ and $R_{2}$ and $\left(u_{1}, u_{2}\right) \in \operatorname{Reg}(R)$. Then the degree of the vertex $\left(u_{1}, u_{2}\right)$ in $\operatorname{Reg}(\Gamma(R))$ is even if and only if $\left|\operatorname{Reg}\left(R_{1}\right)\right|$, $\left|\operatorname{Reg}\left(R_{2}\right)\right|$ are both odd and $\operatorname{deg}_{1}\left(u_{1}\right), \operatorname{deg}_{2}\left(u_{2}\right)$ are both even.

Now, we are ready to prove the following theorem.

3.5. Theorem. Let $R$ be a finite ring. Then $\operatorname{Reg}(\Gamma(R))$ is a regular graph of even degree if and only if $R$ is a field or a product of two or more fields of even orders. 
Proof. Let $R=\prod_{i=1}^{n} R_{i}, n \geq 2$, where $R_{i}$ is a finite local ring for all $i$. First, we will study the three special cases: (i) $|R|$ is odd or (ii) $R_{i}$ is a field of even order for all $i$, or (iii) $R_{i}$ is not a field of even order for all $i$. Using induction in each case, Theorem 3.1 and the above two lemmas, we get $\operatorname{Reg}(\Gamma(R))$ is a regular graph of odd order and even degree when $R$ is a product of fields of even orders, and it is a regular graph of even order and odd degree otherwise. Now, we move to the case where $R$ is a product of fields of even orders and local rings that are not fields of even orders, note that $R \cong S \times T$, where $S$ is the product of all fields $R_{i}^{\prime} s$ and $T$ is the product of all not fields local rings $R_{i}^{\prime} s$. Then $\operatorname{Reg}(\Gamma(R))$ is a regular graph of even order and odd degree. Finally if $|R|=2^{m} t$, where $t>1$ is odd integer, we may write $R \cong S \times T$, where $|S|=2^{m}$, and $|T|=t$. Therefore, $\operatorname{Reg}(\Gamma(R))$ is a regular graph of even order and odd degree.

Note that $Z(\Gamma(R))$ is a regular graph, of degree $|Z(R)|-1$, when $R$ is a local ring since $Z(\Gamma(R)) \cong K_{|Z(R)|}$. However, $Z(\Gamma(R))$ is not regular if $R$ is a product of two rings, since $N((0,0))=Z(R) /\{(0,0)\}$ and $N((0,1)) \subseteq Z(R) /\{(1,0),(0,1)\}$. So, we get the following.

3.6. Theorem. Let $R$ be a finite ring, then

(i) $Z(\Gamma(R))$ is a regular graph if and only if $R$ is a local ring

(ii) $Z(\Gamma(R))$ is a regular graph of even degree if and only if $R$ is a field or $R$ is a local ring of odd order.

3.7. Corollary. $\quad$ (i) $T\left(\Gamma\left(\mathbb{Z}_{n}\right)\right)$, and $\operatorname{Reg}\left(\Gamma\left(\mathbb{Z}_{n}\right)\right)$ are regular graphs of even degrees if and only if $n=2$.

(ii) $Z\left(\Gamma\left(\mathbb{Z}_{n}\right)\right)$ is regular graph of even degree if and only if $n=2$ or $n=p^{m}, p$ is odd prime and $m \geq 1$.

\section{When are $\operatorname{Reg}(\Gamma(R))$ and $Z(\Gamma(R))$ Eulerian?}

A graph is said to be Eulerian if it has a closed trail containing all of its edges. Or equivalently, a connected graph $G$ is Eulerian if and only if the degree of each vertex in $V(G)$ is even.

Clearly, if $R$ is a finite local ring, then $T(\Gamma(R))$ is non Eulerian, and $\operatorname{Reg}(\Gamma(R))$ is Eulerian if and only if $R \cong \mathbb{Z}_{2}$, while $Z(\Gamma(R))$ is Eulerian if and only if $|R|$ is odd or $R$ is a field.

The next theorem, which is due to Shekarriz et al. [16], characterizes Eulerian $T(\Gamma(R))$ when $R$ is a finite ring.

4.1. Theorem. Let $R$ be a finite ring, then the graph $T(\Gamma(R))$ is Eulerian if and only if $R$ is a product of two or more fields of even orders.

Let $R$ be a direct product of two rings. Then $\operatorname{Reg}(\Gamma(R))$ is connected, since for any two vertices $(a, b)$ and $(x, y)$ in $\operatorname{Reg}(\Gamma(R)),(a, b)-(-a,-y)-(x, y)$ is a path joining the two non-adjacent vertices, [1]. So, for any finite non local ring $R, \operatorname{Reg}(\Gamma(R))$ is connected.

Using Theorem 3.5, the following theorem is obtained.

4.2. Theorem. Let $R$ be a finite ring. Then the graph $\operatorname{Reg}(\Gamma(R))$ is Eulerian if and only if $R \cong \mathbb{Z}_{2}$ or $R$ is a product of two or more fields of even orders.

Finally, we investigate when $Z(\Gamma(R))$ is Eulerian.

4.3. Theorem. Let $R$ be a finite ring. Then $Z(\Gamma(R))$ is Eulerian if and only if $R$ is a field or $|R|$ is odd. 
Proof. Clearly, if $R$ is is a local ring, then $Z(\Gamma(R))$ is Eulerian if and only if $R$ is a field or $|R|$ is odd. Suppose that $R=\prod_{i=1}^{n} R_{i}$, where $R_{i}$ is a finite local ring for all $i$. Then we have two cases.

Case 1: $|R|$ is even. If $Z(\Gamma(R))$ is Eulerian, then $\operatorname{deg}((0,0, \ldots, 0))=|Z(R)|-1$ is even. From Lemma 3.3, $R$ is a product of fields of even orders. So $\operatorname{deg}((1,0,0, \ldots, 0)=$ $|Z(R)|-1-\prod_{i=2}^{n}\left|\operatorname{Reg}\left(R_{i}\right)\right|$ is odd, a contradiction.

Case 2: $|R|$ is odd. Then $\left|R_{i}\right|$ is odd for all $i$. Take $w=\left(w_{i}\right) \in Z(R)$. Define $T=\left\{t \in\{1,2, . ., n\}: w_{t} \in Z\left(R_{t}\right)\right\}$ and $J=\{1,2, . ., n\} \backslash T$. Now, to compute the degree of $w$ in $Z(\Gamma(R))$, note that for any finite local ring of odd order $S$, the sum of any two elements is a zero-divisor if and only if both elements are zero-divisors or one of them belongs to the coset $x+Z(S)$ and the other belongs to the coset $-x+Z(S)$, where $x \in \operatorname{Reg}(S)$. So, the vertex $a=\left(a_{i}\right) \in Z(R) \backslash\{w\}$ is non-adjacent to $w$ when $a_{i} \in \operatorname{Reg}\left(R_{i}\right)$ for all $i \in T$, and $\left.a_{i} \in R_{i} \backslash-w_{i}+Z\left(R_{i}\right)\right)$ for all $i \in J$ and $a_{i} \in Z\left(R_{i}\right)$ for some $i \in J$. Since $\left|-w_{i}+Z\left(R_{i}\right)\right|=\left|Z\left(R_{i}\right)\right|$ for all $i$, we have $\operatorname{deg}(w)=(|Z(R)|-1)-$ $\left(\prod_{i \in T}\left|\operatorname{Reg}\left(R_{i}\right)\right|\left(\prod_{i \in J}\left|\operatorname{Reg}\left(R_{i}\right)\right|-\prod_{i \in J}\left(\left|\operatorname{Reg}\left(R_{i}\right)\right|-\left|Z\left(R_{i}\right)\right|\right)\right)\right.$. Since $|Z(R)|$ is odd and $\left|\operatorname{Reg}\left(R_{i}\right)\right|$ is even for all $i$, we get $\operatorname{deg}(w)$ is even. Moreover $Z(\Gamma(R))$ is connected graph since 0 adjacent $\mathrm{s}$ to all other vertices in $Z(\Gamma(R))$. Thus $Z(\Gamma(R))$ is Eulerian.

\subsection{Corollary. $\quad$ (i) $T\left(\Gamma\left(\mathbb{Z}_{n}\right)\right)$ is never Eulerian.}

(ii) $\operatorname{Reg}\left(\Gamma\left(\mathbb{Z}_{n}\right)\right)$ is Eulerian if and only if $n=2$.

(iii) $Z\left(\Gamma\left(\mathbb{Z}_{n}\right)\right)$ is Eulerian if and only if $n=2$ or $n$ is an odd number.

\section{When are $T(\Gamma(R)), \operatorname{Reg}(\Gamma(R))$ and $Z(\Gamma(R))$ locally homogeneous?}

A graph $G$ is called locally homogeneous if the graph induced by the neighborhoods of any two vertices are isomorphic. Let $H$ be a given graph. A graph $G$ is called locally $H$ if for each vertex $v \in V(G)$, the subgraph induced by the open neighborhood of $v, N(v)$, is isomorphic to $H$. Locally $H$ graphs are also called locally homogeneous [17]. Graphs associated with algebraic structures are known to exhibit some symmetrical properties, see for example [17]. In this section, we investigate homogeneity in the total graphs associated with rings.

Let $R$ be a local ring with $|Z(R)|=\alpha$. Then by Theorem 1.1, $T(\Gamma(R))$ is locally $H$ if and only if $2 \in Z(R)$. In this case, $H=K_{\alpha-1}$. So, if $R$ is a finite local ring, then $T(\Gamma(R))$ is locally $H$ if and only if $|R|$ is even, $\operatorname{Reg}(\Gamma(\mathrm{R}))$ is either locally $K_{\alpha-1}$ or $\overline{K_{\alpha}}$, and $Z(\Gamma(R))$ is locally $K_{\alpha-1}$. The next theorem treats the case for any finite ring $R$.

5.1. Theorem. Let $R$ be a finite ring. Then

(i) Let $x$ and $y$ be two distinct vertices in $T(\Gamma(R))$. Then, the subgraph of $T(\Gamma(R))$ induced by $N(x)$ is isomorphic to the subgraph induced by $N(y)$ if and only if $|R|$ is even.

(ii) Let $x$ and $y$ be two distinct vertices in $\operatorname{Reg}(\Gamma(R))$. Then, the subgraph of $\operatorname{Reg}(\Gamma(R))$ induced by $N(x)$ is isomorphic to the subgraph induced by $N(y)$.

(iii) $Z(\Gamma(R))$ is locally $H$ if and only if $R$ is a local ring. In this case, $H=K_{|Z(R)|-1}$.

Proof. (1) If $|R|$ is odd, then $2 \notin Z(R)$, and so, $T(\Gamma(R)$ is not regular, hence we may assume that $|R|$ is even. Let $R=\prod_{i=1}^{n} R_{i}$. Where each $R_{i}$ is a local ring. Without loss of generality, we may assume that $2 \in Z\left(R_{1}\right)$. Obviously, for $n=1$, the result holds. If $S=\Pi_{i=2}^{n} R_{i}$, then $R=R_{1} \times S$. We will prove that the neighborhoods of any two distinct vertices in $T(\Gamma(R))$ are isomorphic. Let $(a, b)$ be an arbitrary element in $R$. Let $N_{1}=\{a\} \times(S /\{b\}), N_{2}=\left\{(x, y) \in R: x \neq a, x+a \in Z\left(R_{1}\right)\right\}$ and $N_{3}=\left\{(x, y) \in R: x+a \in \operatorname{Reg}\left(R_{1}\right)\right.$, and $\left.y+b \in Z(S)\right\}$. Note that $N_{1}, N_{2}$ and $N_{3}$ form a 
partition for $N((a, b))$. Thus $N((a, b))=N_{1} \cup N_{2} \cup N_{3} . N_{1}$ induces a complete graph of order $|S|-1$. For each fixed vertex $r \in S$, let $N_{2_{r}}=\left\{(x, r) \in R: x \neq a, x+a \in Z\left(R_{1}\right)\right\}$. Each set $N_{2_{r}}$ induces a copy of the graph induced by $N(a)$ in the graph $T\left(\Gamma\left(R_{1}\right)\right.$ which a complete graph. Besides, for each pair of distinct vertices in $r, s \in S$, each vertex $\left(x_{1}, r\right)$ in $N_{2_{r}}$ is adjacent to each vertex $\left(x_{2}, s\right)$ in $N_{2_{s}}$, since $x_{1}+x_{2}+2 a \in Z\left(R_{1}\right)$ implies that $x_{1}+x_{2} \in Z\left(R_{1}\right)$. Each vertex in $N_{1}$ is adjacent to each vertex in $N_{2}$.

Now, we claim that $N_{3}$ induces a complete graph. Let $\left(x_{1}, y_{1}\right),\left(x_{2}, y_{2}\right) \in N_{3}$ then $a+x_{1} \in \operatorname{Reg}\left(R_{1}\right)$ and $a+x_{2} \in \operatorname{Reg}\left(R_{1}\right)$. we study two cases:

Case 1: $a \in Z\left(R_{1}\right)$, then both $x_{1}$ and $x_{2}$ belong to $\operatorname{Reg}\left(R_{1}\right)$. By Theorem 2.9 of [1], $x_{1}+x_{2} \in Z\left(R_{1}\right)$ or $x_{1}-x_{2} \in Z\left(R_{1}\right)$. Assume that $x_{1}-x_{2} \in Z\left(R_{1}\right)$, say $x_{1}-x_{2}=z$ and $x_{1}+x_{2}=r$, for some $r \in \operatorname{Reg}\left(R_{1}\right)$ and some $z \in Z\left(R_{1}\right)$. This implies that $2 x_{1}-z=r$ which is a contradiction, thus $x_{1}+x_{2} \in Z\left(R_{1}\right)$ and hence $\left(x_{1}, y_{1}\right)$ is adjacent to $\left(x_{2}, y_{2}\right)$.

Case 2. $a \in \operatorname{Reg}\left(R_{1}\right)$, we have $x_{1}+a=r_{1}$ and $x_{2}+a=r_{2}$, where $r_{1}, r_{2} \in \operatorname{Reg}\left(R_{1}\right)$. Either $r_{1}+r_{2} \in Z\left(R_{1}\right)$ or $r_{1}-r_{2} \in Z\left(R_{1}\right)$. If $r_{1}+r_{2} \in Z\left(R_{1}\right)$, then $x_{1}+x_{2}+2 a \in Z\left(R_{1}\right)$, and hence $x_{1}+x_{2} \in Z\left(R_{1}\right)$. If $r_{1}-r_{2} \in Z\left(R_{1}\right)$, then $x_{1}-x_{2} \in Z\left(R_{1}\right)$, if $x_{1} \in \operatorname{Reg}\left(R_{1}\right)$, then $x_{1}-a=z_{1}$, for some $z_{1} \in Z\left(R_{1}\right)$. But $x_{1}+a=r_{1}$, where $r_{1} \in \operatorname{Reg}\left(R_{1}\right)$. So, $2 x_{1}=z_{1}+r_{1}$ which is a contradiction. Similarly, $x_{2} \in Z\left(R_{1}\right)$, and hence, $\left(x_{1}, y_{1}\right)$ is adjacent to $\left(x_{2}, y_{2}\right)$.

If a vertex $\left(x_{1}, y_{1}\right) \in N_{2}$, is adjacent to a vertex $\left(x_{2}, y_{2}\right) \in N_{3}$, then, $x_{1}+x_{2} \in \operatorname{Reg}\left(R_{1}\right)$, To see this write $x_{1}+a=z$ and $x_{2}+a=r$, where $z \in Z\left(R_{1}\right)$ and $r \in \operatorname{Reg}(S)$, this implies that $x_{1}+x_{2}+(2 a-z)=r$, and so, $x_{1}+x_{2} \in \operatorname{Reg}\left(R_{1}\right)$. We may write $Z(S)=\bigcup_{i=1}^{m} I_{i}$, where each $I_{i}$ is a maximal ideal of $S$. Suppose that $b \in b_{i}+I_{i}$, if $a_{i}+b_{i} \in I_{i}$, then $y_{2} \in \bigcup_{i=1}^{m} a_{i}+I_{i}$. Let $G$ be the bipartite subgraph of $T(\Gamma(R))$ with partite sets $N_{2}$ and $N_{3}$ where two vertices $\left(x_{1}, y_{1}\right) \in N_{2}$ and $\left(x_{2}, y_{2}\right) \in N_{3}$ are adjacent if $y_{1}+y_{2} \in Z(S)$. Similarly, $N_{1} \cup N_{3}$ with edges joining $N_{1}$ to $N_{3}$ form another bipartite graph. Finally, since this description of $N((a, b))$ does not depend on the choice of $(a, b)$, we conclude that the neighborhood of any two vertices in $T(\Gamma(R))$ are isomorphic .

(ii) Considering Theorem 3.2, $\operatorname{Reg}(\Gamma(R))$ is regular. Let $R=\prod_{i=1}^{n} R_{i}$. For $i=$ $1,2, \ldots, n$, let $G_{i}$ be the spanning subgraph of $\operatorname{Reg}(\Gamma(R))$ where two vertices $\left(x_{1}, x_{2}, \ldots x_{n}\right)$ and $\left(y_{1}, y_{2}, \ldots y_{n}\right)$ are adjacent in $G_{i}$ if $x_{i}+y_{i} \in Z\left(R_{i}\right)$. The graph $\operatorname{Reg}(\Gamma(R))$ is the overlay of the layers $G_{i}, i=1,2, \ldots, n$. Each layer is a union of complete graphs or a union of complete bipartite graphs. Let $x$ and $y$ be two distinct vertices in $\operatorname{Reg}(\Gamma(R))$. Let $N_{i}(x)$ and $N_{i}(y)$ be the open neighborhoods of $x$ and $y$ respectively, in the graph $G_{i}$. Then $N(x)=\cup_{i=1}^{i=n} N_{i}(x)$, and $N(y)=\cup_{i=1}^{i=n} N_{i}(y)$. So, $N(x)$ is the overlay of the layers induced by $N_{i}(x), i=1,2, \ldots, n$. Similar result holds for $N(y)$. Observe that for each $i=1,2, \ldots, n, N_{i}(x)$ and $N_{i}(y)$ induce isomorphic subgraphs of the graph $G_{i}$, consequently, $N(x)$ and $N(y)$ induce isomorphic subgraphs of the graph $\operatorname{Reg}(\Gamma(R))$.

(iii) Direct result of Theorem 3.6 part (1) and the argument before Theorem 6.1.

\section{References}

[1] Anderson, D. F., Badawi, A. The Total graph of a commutative ring, J. Algebra 320, 2706-2719, (2008).

[2] Anderson, D. F., Badawi, A.The gereralized total graph of a commutative ring, J. Algebra App. 12 (2013), doi: 10.1142/S021949881250212X (2013).

[3] Akbari, S., Kiani, D., Mohammadi, F., Moradi, S. The total graph and regular graph of a commutative ring. J. Pure Appl. Algebra 213 (12) 2224-2228 (2009).

[4] Akbari, S., Haydari, F. The regular graph of a commutative ring, Period Math. Hungar. 67 (2) 211-220 (2013).

[5] Asir, T., Chelvam, T. On the total graph and its complement of a commutative ring, Comm. Algebra. 41, 38820-3835, dio:10.1080/009282.2012.678956 (2013). 
[6] Badawi, A. On the total graph of a ring and its related graphs: A Survey, M. Fontana et al. (eds.), Commutative Algebra: Recent Advances in Commutative Rings, Integer-Valued Polynomials, and Polynomial Functions, Springer, New York. doi: 10.1007/978-1-49390925-4-3 (2014).

[7] Chelvam, T., Asir, T. Domination in the total graph of a commutative ring, J. Combin. Math. Combin. Comput. 87, 147-158 (2013).

[8] Erić, A. Lj., Pucanović, Z. S. Some properties of the line graphs associated to the total graph of a commutative ring, Pure Appl. Math. J. 2 (2) 51-55 (2013).

[9] Harary, F. Graph Theory. Publishing Co., Reading Mass (1972).

[10] Nazzal K. Total graphs associated to a commutative ring, Palest. J. Math. 5 (Spec.1) (2016).

[11] Koshy, T. Elementary number theory with applications. Harcourt Academic press Co.(2002).

[12] Maimani, H. R., Wickham, C. Yassemi, S. Rings whose total graphs have genus at most one, Rocky Mountain J. Math. 42, 1421-1758 (2010).

[13] Pucanovi, Z., Petrovi, Z. On the radius and the relation between the total graph of a commutative ring and its extensions, Pub. Ins. Math. (Beograd) (N.S.) 89,1-9 (2011).

[14] Ramin, A. The total graph of a finite commutative ring. Turk J. Math, 37, 391-397 (2013).

[15] Sander, T., Nazzal, K. Minimum flows in the total graph of a finite commutative ring, Trans. Comb. 3(3), 11-20 (2014).

[16] Shekarriza, M. H. Shirdareh Haghighia, M. H., Sharifa, H. On the total graph of a finite commutative ring, Comm. Algebra. 40, 2798-2807 (2012).

[17] Vince A. Locally homogeneous graphs from groups, J. Graph Theory, 4, 417-422 (1981). 